\title{
ENCODING NEUROANATOMICAL INFORMATION USING WEIGHTED SPHERICAL HARMONIC REPRESENTATION
}

\author{
Moo K. Chung ${ }^{1,2}$, Kim M. Dalton ${ }^{2}$, Richard J. Davidson ${ }^{3}$ \\ ${ }^{1}$ Department of Biostatistics and Medical Informatics \\ ${ }^{2}$ Waisman Laboratory for Brain Imaging and Behavior \\ ${ }^{3}$ Department of Psychology and Psychiatry \\ University of Wisconsin-Madison
}

\begin{abstract}
Cortical surfaces can be modeled using the recently developed weighed spherical harmonic (SPHARM) representation. The weighted-SPHARM representation incorporates data smoothing, parametrization and surface registration in a unified mathematical framework. The weighted-SPHARM represents the surface coordinates as a weighted linear combination of spherical harmonics in such a way that it solves a heat equation. The representation can decomposes an arbitrary surface into symmetric and asymmetric parts with respect to mirror reflection. By analyzing this unique decomposition, surface asymmetry can be quantified. We have applied our technique in quantifying abnormal brain asymmetry in autistic subjects.
\end{abstract}

Index Terms - Spherical Harmonics, Cortical Surface, Mirror Symmetry, Heat diffusion, SPHARM representation

\section{INTRODUCTION}

We propose a novel 2D surface modeling framework called the weighted spherical harmonic representation (SPHARM) that can be used in encoding cortical shape information. The novelty of our proposed method is that cortical surface parameterization, surface registration, surface data smoothing and cortical surface asymmetry analysis can be performed within a single unified mathematical framework providing a more consistent modeling and validation approach than traditionally available. The weighted-SPHARM representation is a spectral method [9], where a linear combination explicit basis functions is used to represent noisy cortical surface data. The basis expansion corresponds to the solution of an isotropic heat equation on a unit sphere. Unlike the previous heat diffusion related smoothing that solves the heat equation nonparametrically [1] [3] [4], the result of the weighted-SPHARM is explicitly given as a weighted linear combination of spherical harmonics. This provides a more natural setting for statistical modeling. The representation can be further used in surface registration that reduces the improper alignment of sulcal folding patterns between subjects and across hemispheres within a subject.
In this paper, we show how this new representation can be used in a hemisphere asymmetry analysis. Using the inherent angular symmetry in the spherical harmonics, the traditional asymmetry index of the form $(\mathrm{L}-\mathrm{R}) /(\mathrm{L}+\mathrm{R})$ [11] can be expressed as the ratio between the sum of positive and negative order harmonics. This asymmetry index is used as a response variable in a statistical model in quantifying abnormal asymmetric pattern in 16 autistic subjects.

\section{METHODS}

An outer cortical surface $\mathcal{M}$ is assumed to be a smooth 2D Riemannian manifold topologically equivalent to a unit sphere [8] [5]. The outer cortical surface is obtained from a $\mathrm{T}_{1}$ weighted magnetic resonance image using a deformable surface algorithm [12] and represented as a triangle mesh consisting of 40,962 vertices and 81,920 triangles (Figure 1). The algorithm starts with a spherical mesh and deforms to fit the shape of the cortex guaranteeing the same topology. By following the trajectory of the deformation, we can establish a smooth bijective mapping between $\mathcal{M}$ and a unit sphere $S^{2}$. Based on this mapping, we parameterize the Cartesian coordinates $v=\left(v_{1}, v_{2}, v_{3}\right)$ of $\mathcal{M}$ with the polar angle $\theta \in[0, \pi]$ and the azimuthal angle $\varphi \in[0,2 \pi)$ so that each component $v_{i}$ can be expressed as

$$
v_{i}=v_{i}(\theta, \varphi) .
$$

As a convention, the plane $v_{2}=0$ is chosen to be the midsagittal cross-section that separates the two hemispheres of the brain (Figure 2). The area defined by $0<\varphi<\pi$ is the left hemisphere.

\subsection{Weighted Spherical Harmonic Representation}

The spherical harmonic $Y_{l m}$ of degree $l$ and order $m$ [5] [6] is defined as

$Y_{l m}=\left\{\begin{array}{cc}c_{l m} P_{l}^{|m|}(\cos \theta) \sin (|m| \varphi), & -l \leq m \leq-1, \\ \frac{c_{l m}}{\sqrt{2}} P_{l}^{|m|}(\cos \theta), & m=0, \\ c_{l m} P_{l}^{|m|}(\cos \theta) \cos (|m| \varphi), & 1 \leq m \leq l,\end{array}\right.$ 

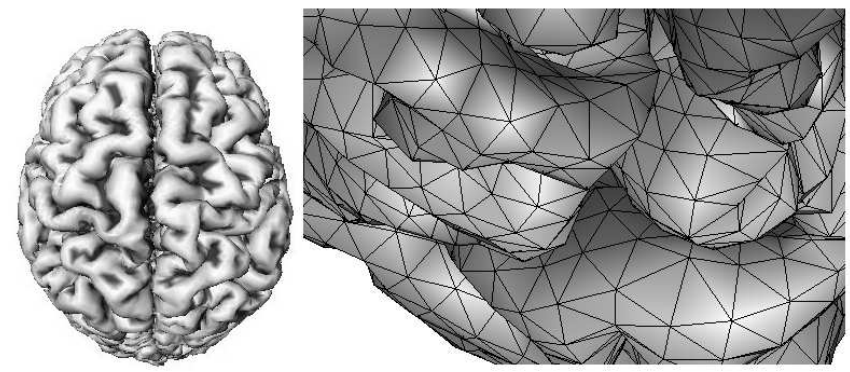

Fig. 1. Left: Outer cortical surface obtained from a deformable surface algorithm [12]. Right: A close up image of the outer cortical surface represented as a triangle mesh consisting of 40962 vertices. The cortical thickness measures are obtained at the mesh vertices.

where

$$
c_{l m}=\sqrt{\frac{2 l+1}{2 \pi} \frac{(l-|m|) !}{(l+|m|) !}}
$$

and $P_{l}^{m}$ is the associated Legendre polynomial of order $m$. The spherical harmonics are orthonormal with respect to the inner product

$$
\left\langle f_{1}, f_{2}\right\rangle=\int_{S^{2}} f_{1}(\theta, \varphi) f_{2}(\theta, \varphi) \sin \theta d \theta d \varphi
$$

Surface coordinates $v_{i}$ will be modeled independently as

$$
v_{i}(\theta, \varphi)=h_{i}(\theta, \varphi)+\epsilon_{i}(\theta, \varphi),
$$

where $h_{i}$ is the unknown smooth coordinate function to be estimated and $\epsilon_{i}$ is zero mean random fields, possibly Gaussian. Functional measurements obtained along the cortical surface will be modeled similarly. Traditionally, the coordinate functions $h_{i}$ has been estimated using the SPHARM representation [2] [13] [14]:

$$
\widehat{h_{i}}(\theta, \varphi)=\sum_{l=0}^{k} \sum_{m=-l}^{l}\left\langle v_{i}, Y_{l m}\right\rangle Y_{l m}(\theta, \varphi) .
$$

The weakness of representation (2) is that it generates the Gibbs phenomenon (ringing artifact) [5] [10] for discontinuous or rapidly changing functional measurements. The Gibbs phenomenon can be effectively removed if the expansion (2) converges faster as the degree $k$ goes to infinity. By weighting the SPHARM coefficients exponentially smaller, we can make the representation converges faster. This can be achieved as follows.

Define heat kernel smoothing [4] [5] of coordinate function $v_{i}$ as

$$
K_{\sigma} * v_{i}(\theta, \varphi)=\int_{S^{2}} K_{\sigma}\left(\theta, \varphi, \theta^{\prime}, \varphi^{\prime}\right) v_{i}\left(\theta^{\prime}, \varphi^{\prime}\right) \sin \theta^{\prime} d \theta^{\prime} d \varphi^{\prime}
$$
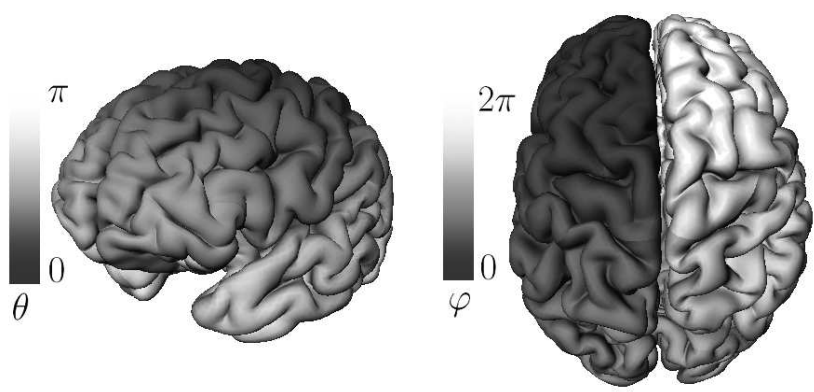

Fig. 2. Parameterization of cortical surface using the spherical coordinate system. The north and south poles are chosen in the plane, i.e. $v_{2}=0$, that separates the left and the right hemispheres.

On unit sphere, heat kernel $K_{\sigma}$ is explicitly given as

$$
K_{\sigma}\left(\theta, \varphi, \theta^{\prime}, \varphi^{\prime}\right)=\sum_{l=0}^{\infty} \sum_{m=-l}^{l} e^{-l(l+1) \sigma} Y_{l m}(\theta, \varphi) Y_{l m}\left(\theta^{\prime}, \varphi^{\prime}\right) .
$$

The parameter $\sigma$ controls the dispersion of the kernel so we will simply call it as the bandwidth. Then using the explicit expression for the heat kernel, heat kernel smoothing can be rewritten as

$$
K_{\sigma} * v_{i}(\theta, \varphi)=\sum_{l=0}^{\infty} \sum_{m=-l}^{l} e^{-l(l+1) \sigma}\left\langle v_{i}, Y_{l m}\right\rangle Y_{l m}(\theta, \varphi) .
$$

The $k$-th degree expansion of heat kernel smoothing will be called the $k$-th degree weighted-SPHARM representation:

$$
\widehat{h_{i}}(\theta, \varphi)=\sum_{l=0}^{k} \sum_{m=-l}^{l} e^{-l(l+1) \sigma}\left\langle v_{i}, Y_{l m}\right\rangle Y_{l m}(\theta, \varphi)
$$

and it will be taken as our estimate for the unknown coordinate function $h_{i}$. The weighted-SPHARM converges to the solution of isotropic heat diffusion

$$
\frac{\partial g}{\partial \sigma}=\Delta g, g(\theta, \varphi, \sigma=0)=v_{i}(\theta, \varphi)
$$

as $k$ goes to infinity.

\subsection{Iterative Residual Fitting Algorithm}

The SPHARM coefficients are estimated based on the iterative procedure that utilizes the orthonormality of the spherical harmonics. The coordinate functions $v_{i}$ are measured at $n=40962$ mesh vertices $\left(\theta_{j}, \varphi_{j}\right)$ [12]. Then we have the following set of linear equations:

$$
v_{i}\left(\theta_{j}, \varphi_{j}\right)=\sum_{l=0}^{k} \sum_{m=-l}^{l}\left\langle v_{i}, Y_{l m}\right\rangle Y_{l m}\left(\theta_{j}, \varphi_{j}\right)
$$


Previously the coefficients $\left\langle v_{i}, Y_{l m}\right\rangle$ have been estimated by inverting the system of linear equations in the least squares fashion [5] [13] [14]. However, for more than 40000 mesh vertices used in our study, the size of the the linear system (4) can easily reach the RAM memory limit of the most personal computers considering the degree $k$ can be anywhere between 1 and 90 . To address this problem, we have developed a new numerical technique called the iterative residual fitting (IRF) algorithm [5] [13]. In IRF, the coefficients are estimated iteratively by breaking the large least squares problem (4) into smaller least squares problems.

At the 0-th degree, the coefficient $\left\langle v_{i}, Y_{00}\right\rangle$ is estimated by solving a smaller least squares problem

$$
v_{i}\left(\theta_{j}, \varphi_{j}\right)=\left\langle v_{i}, Y_{00}\right\rangle Y_{00}\left(\theta_{j}, \varphi_{j}\right) .
$$

This servers as the initial condition for the IRF algorithm. Then iteratively at the $l$-th degree, the set of $2 l+1$ coefficients $\left\langle v_{i}, Y_{l,-1}\right\rangle, \cdots,\left\langle v_{i}, Y_{l,-1}\right\rangle$ are estimated by solving

$$
r_{i}^{l}\left(\theta_{j}, \varphi_{j}\right)=\sum_{m=-l}^{l}\left\langle v_{i}, Y_{l m}\right\rangle Y_{l m}\left(\theta_{j}, \varphi_{j}\right)
$$

The $l$-th residual $r_{i}^{l}$ is given by

$$
\left.r_{i}^{l}\left(\theta_{j}, \varphi_{j}\right)=v_{i}\left(\theta_{j}, \varphi_{j}\right)-\sum_{l^{\prime}=0}^{l-1} \sum_{m=-l^{\prime}}^{l^{\prime}} \widehat{\left\langle v_{i}, Y_{l m}\right.}\right\rangle Y_{l m}\left(\theta_{j}, \varphi_{j}\right),
$$

where $\left\langle\widehat{v_{i}, Y_{l m}}\right\rangle$ is the estimation obtained in the previous degrees. The stopping rule for the iteration is when the change of the root mean squared errors (RMSE) i.e.

$$
\mathbf{R M S E}=\left[\frac{1}{n} \sum_{j=1}^{n} r_{i}^{k}\left(\theta_{j}, \varphi_{j}\right)\right]^{1 / 2}
$$

is no longer significant (Figure 2.2). The MATLAB implementation is publically available at

www. stat. wisc. edu/ mchung/softwares

/weighted-SPHARM/weighted-SPHARM.html.

The numerical accuracy and validation issues are addressed in [5] [13].

\subsection{SPHARM-correspondence}

Given the weighted-SPHARM representation, we need to establish the correspondence between hemispheres and between subjects. Consider a weighted-SPHARM surface $\widehat{h_{i}}$ obtained from coordinate functions $v_{i}$ as in equation (3). Consider the other weighted-SPHARM surface $\widehat{j_{i}}$ obtained from coordinate functions $w_{i}$ :

$$
\widehat{j}_{i}(\theta, \varphi)=\sum_{l=0}^{k} \sum_{m=-l}^{l} e^{-l(l+1) \sigma}\left\langle w_{i}, Y_{l m}\right\rangle(\theta, \varphi) .
$$

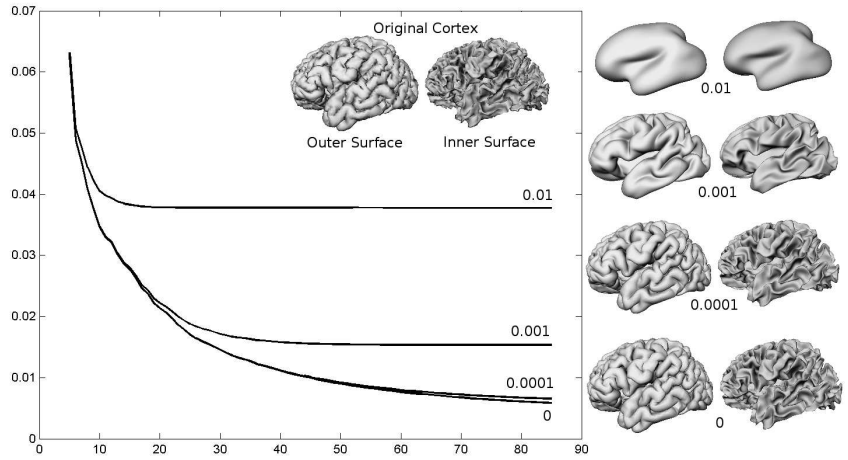

Fig. 3. Plots of the RMSE of the weighted-SPHARM for various bandwidth $\sigma(0.01,0.001,0.0001,0)$ over degree $k$. As the degree increases, at certain point, the change of RMSE is no longer statistically significant and this is where the optimal degree is selected. The surfaces are the weighted-SPHARM at the 85-th degree.

Suppose the surface $\widehat{h_{i}}$ is deformed to $\widehat{h_{i}}+d_{i}\left(\widehat{h_{i}}\right)$, where $d_{i}$ is the displacement vector field. The optimal displacement that minimizes the discrepancy between $\widehat{h}_{i}+d_{i}\left(\widehat{h}_{i}\right)$ and $\widehat{j}_{i}$ in the least squares fashion [5] is given by

$$
\widehat{d}_{i}(\theta, \varphi)=\sum_{l=0}^{k} \sum_{m=-l}^{l} e^{-l(l+1) \sigma}\left(w_{l m}^{i}-v_{l m}^{i}\right) Y_{l m}(\theta, \varphi) .
$$

The optimal displacement is simply obtained by taking the difference between two weighted-SPHARM representation. Then a point $\widehat{h_{i}}\left(\theta_{0}, \varphi_{0}\right)$ in one surface corresponds to a point $\widehat{j_{i}}\left(\theta_{0}, \varphi_{0}\right)$ in the other surface. We will refer this surface correspondence as the SPHARM-correspondence [5].

The SPHARM-correspondence is further used to establish the inter-hemispheric correspondence by letting $\widehat{j_{i}}$ to be the mirror reflection of $\widehat{h_{i}}$. The mirror reflection of $\widehat{h_{i}}$ with respect to the mid-sagittal cross section, i.e. $v_{2}=0$, is given by

$$
\widehat{j_{i}}(\theta, \varphi)=\widehat{h}_{i}^{*}(\theta, \varphi)=\widehat{h}_{i}(\theta, 2 \pi-\varphi),
$$

where ${ }^{*}$ denotes the mirror reflection. The point $\widehat{h}_{i}\left(\theta_{0}, \varphi_{0}\right)$ in the left hemisphere will be mirror reflected to $\widehat{j}_{i}\left(\theta_{0}, 2 \pi-\varphi_{0}\right)$ in the right hemisphere. Then $\widehat{j}_{i}\left(\theta_{0}, 2 \pi-\varphi_{0}\right)$ is matched to $\widehat{h}_{i}\left(\theta_{0}, 2 \pi-\varphi_{0}\right)$ in the SPHARM-correspondence sense. Hence, the point $\widehat{h}_{i}\left(\theta_{0}, \varphi_{0}\right)$ in the left hemisphere corresponds to the point $\widehat{h}_{i}\left(\theta_{0}, 2 \pi-\varphi_{0}\right)$ in the right hemisphere ( Figure 4).

\subsection{Encoding Surface Asymmetry Information}

The inter-hemispheric correspondence is used to compare cortical thickness measurements across the hemispheres. Cortical thickness [3] [12], which measures the thickness of gray 

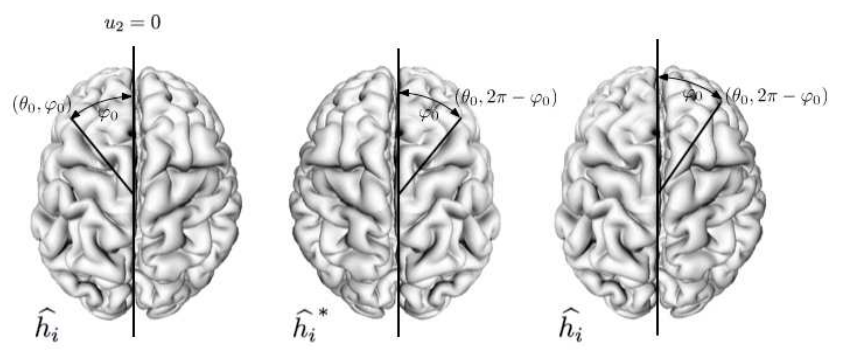

Fig. 4. The point $\widehat{h}_{i}\left(\theta_{0}, \varphi_{0}\right)$ (left) corresponds to $\widehat{h}_{i}^{*}(\theta, 2 \pi-$ $\left.\varphi_{0}\right)$ (middle) after mirror reflection with respect to the $u_{2}=$ 0 . From the SPHARM-correspondence, $\widehat{h}_{i}^{*}\left(\theta, 2 \pi-\varphi_{0}\right)$ matches $\widehat{h}_{i}\left(\theta, 2 \pi-\varphi_{0}\right)$ (right). This establish the mapping from the left hemisphere to the right hemisphere in the least squares fashion.

matter along a cortical surface, is modeled as

$$
f(\theta, \varphi)=g(\theta, \varphi)+\epsilon(\theta, \varphi)
$$

where $g$ is the unknown cortical thickness and $\epsilon$ is a mean zero random field. The weighted-SPHARM representation of cortical thickness $f$ is given by

$$
\widehat{g}(\theta, \varphi)=\sum_{l=0}^{k} \sum_{m=-l}^{l} e^{-l(l+1) \sigma}\left\langle f, Y_{l m}\right\rangle Y_{l m}(\theta, \varphi) .
$$

The cortical thickness $\widehat{g}\left(\theta_{0}, \varphi_{0}\right)$ at point $\widehat{h}_{i}\left(\theta_{0}, \varphi_{0}\right)$ in the left hemisphere is matched to the cortical thickness $\widehat{g}\left(\theta_{0}, 2 \pi-\right.$ $\left.\varphi_{0}\right)$ at point $\widehat{h}_{i}\left(\theta_{0}, 2 \pi-\varphi_{0}\right)$ in the right hemisphere. Then using the symmetric and asymmetric properties of spherical harmonics

$$
Y_{l m}(\theta, 2 \pi-\varphi)=\left\{\begin{array}{cc}
-Y_{l m}(\theta, \varphi), & -l \leq m \leq-1, \\
Y_{l m}(\theta, \varphi), & 0 \leq m \leq l,
\end{array}\right.
$$

we obtain

$$
\begin{aligned}
\widehat{g}\left(\theta_{0}, 2 \pi-\varphi_{0}\right)= & \sum_{l=0}^{k} \sum_{m=-l}^{-1} e^{-l(l+1) \sigma}\left\langle f, Y_{l m}\right\rangle Y_{l m}\left(\theta_{0}, \varphi_{0}\right) \\
& -\sum_{l=0}^{k} \sum_{m=0}^{l} e^{-l(l+1) \sigma}\left\langle f, Y_{l m}\right\rangle Y_{l m}\left(\theta_{0}, \varphi_{0}\right) .
\end{aligned}
$$

Comparing it with the expansion for $\widehat{g}\left(\theta_{0}, \varphi_{0}\right)$ in (6), we see that the negative order terms are invariant while the positive order terms change the sign under mirror reflection. Hence we define the symmetry index as

$$
S(\theta, \varphi)=\sum_{l=0}^{k} \sum_{m=-l}^{-1} e^{-l(l+1) \sigma}\left\langle f, Y_{l m}\right\rangle Y_{l m}\left(\theta_{0}, \varphi_{0}\right)
$$
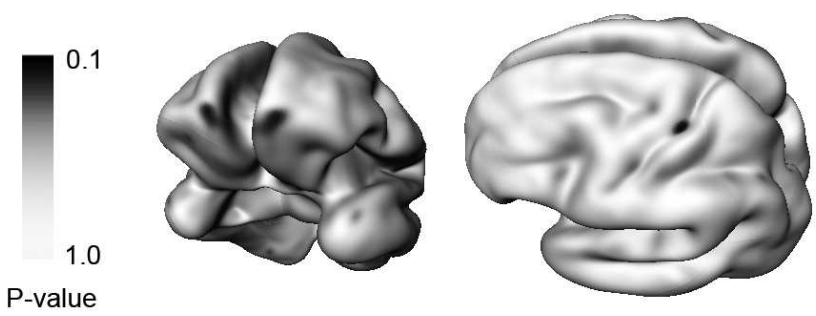

Fig. 5. The statistically significant regions of cortical asymmetry thresholded at the P-value of 0.1 . The P-value has been corrected for multiple comparisons.

while the asymmetry index as

$$
A(\theta, \varphi)=\sum_{l=0}^{k} \sum_{m=0}^{l} e^{-l(l+1) \sigma}\left\langle f, Y_{l m}\right\rangle Y_{l m}\left(\theta_{0}, \varphi_{0}\right) .
$$

The normalize asymmetry index of the form $(\mathrm{L}-\mathrm{R}) /(\mathrm{L}+\mathrm{R})$ is then defined as

$$
N(\theta, \varphi)=\frac{\sum_{l=1}^{k} \sum_{m=-l}^{-1} e^{-1(l+1) \sigma}\left\langle f, Y_{l m}\right\rangle Y_{l m}(\theta, \varphi)}{\sum_{l=0}^{k} \sum_{m=0}^{l} e^{-l(l+1) \sigma}\left\langle f, Y_{l m}\right\rangle Y_{l m}(\theta, \varphi)}
$$

The numerator is the sum of all negative order harmonics while the denominator is the sum of all positive and the 0th order harmonics. Note that $N(\theta, 0)=N(\theta, \pi)=0$. This index is intuitively interpreted as the normalized difference between cortical thickness in the left and the right hemispheres. Note that the larger the value of the index, the larger the amount of asymmetry. The index is invariant under the affine scaling so it is not necessary to control for the global brain size difference in statistical analyses.

\section{APPLICATION}

Three Tesla $\mathrm{T}_{1}$-weighted MR scans were acquired for age matched 16 high functioning autistic and 12 control righthanded males. The autistic subjects were diagnosed by a trained and certified psychologist at the Waisman center at the University of Wisconsin [7]. The $\mathrm{T}_{1}$-weighted images went through various image processing steps, which are described in detail in [5]. Once we obtained the outer cortical surfaces of 28 subjects, the weighted-SPHARM representations $\widehat{h}_{i}$ and the corresponding cortical thickness $\widehat{g}$ are constructed. We have used bandwidth $\sigma=0.001$ corresponding to $k=42$ degrees. For each subject, its normalized asymmetry index $N(\theta, \varphi)$ is computed and modeled as a Gaussian random field. We test the null hypothesis that $N(\theta, \varphi)$ is identical to the both groups for all points $(\theta, \varphi)$ while the alternate hypothesis is that there exists a specific point $\left(\theta_{0}, \varphi_{0}\right)$, at which $N\left(\theta_{0}, \varphi_{0}\right)$ is different for the both groups. The test 
statistic is a $T$ random field, denoted as $T(\theta, \varphi)$. For the multiple comparison correction, we have used the random field theory based approach [15], where the corrected P-value is computed as

$$
P\left[\sup _{(\theta, \varphi) \in S^{2}} T(\theta, \varphi)>h\right] \approx \sum_{d=0}^{2} R_{d}\left(S^{2}\right) \mu_{d}(h) .
$$

In equation (7), $R_{d}$ is the $d$-dimensional Resels of $S^{2}$ and $\rho_{d}$ is the $d$-dimensional Euler characteristic (EC) density of the $T$-field with the 26 degrees of freedom. The computed $\mathrm{P}$-values are projected on the average cortical surface (Figure 5). The average cortical surface is constructed by averaging the SPHARM coefficients of all subjects. The average surface serves as an anatomical landmark for displaying these indices. We found that the central sulci and the prefrontal cortex exhibits abnormal cortical asymmetry pattern in autistic subjects (corrected P-value $<0.1$ ).

\section{REFERENCES}

[1] A. Andrade, F Kherif, J. Mangin, K.J. Worsley, A. Paradis, O. Simon, S. Dehaene, D. Le Bihan, and J-B. Poline. Detection of fmri activation using cortical surface mapping. Human Brain Mapping, 12:79-93, 2001.

[2] C. Brechbuhler, G. Gerig, and O. Kubler. Parametrization of closed surfaces for $3 \mathrm{~d}$ shape description. Computer Vision and Image Understanding, 61:154-170, 1995.

[3] M.K. Chung, K.J. Worsley, S. Robbins, T. Paus, Taylor, J.N. J., Giedd, J.L. Rapoport, and A.C. Evans. Deformation-based surface morphometry applied to gray matter deformation. NeuroImage, 18:198-213, 2003.

[4] M.K. Chung, S. Robbins, Davidson R.J. Alexander A.L. Dalton, K.M., and A.C. Evans. Cortical thickness analysis in autism with heat kernel smoothing. NeuroImage, 25:1256-1265, 2005.

[5] M.K. Chung, L. Shen, K.M. Dalton, A.C. Evans, and R.J. Davidson. Weighted fourier representation and its application to quantifying the amount of gray matter. IEEE Transactions on Medical Imaging, 26:566-581, 2007.

[6] R. Courant and D. Hilbert. Methods of Mathematical Physics: Volume II. Interscience, New York, english edition, 1953.

[7] K.M. Dalton, B.M. Nacewicz, T. Johnstone, H.S. Schaefer, M.A. Gernsbacher, H.H. Goldsmith, A.L. Alexander, and R.J. Davidson. Gaze fixation and the neural circuitry of face processing in autism. Nature Neuroscience, 8:519-526, 2005.
[8] C. Davatzikos and R.N. Bryan. Using a deformable surface model to obtain a shape representation of the cortex. Proceedings of the IEEE International Conference on Computer Vision, 9:2122-2127, 1995.

[9] B. Fornberg. A practical guide to pseudospectral methods Cambridge University Press, 1998.

[10] A. Gelb. The resolution of the gibbs phenomenon for spherical harmonics. Mathematics of Computation, 66:699-717, 1997.

[11] D. Kennedy, K. O’Craven, B. Ticho, A. Goldstein, N. Makris, and J. Henson. Structural and functional brain asymmetries in human situs inversus totalis. $\mathrm{Neu}$ rology, 53:1260-1265, 1999

[12] J.D. MacDonald, N. Kabani, D. Avis, and A.C. Evans. Automated 3-d extraction of inner and outer surfaces of cerebral cortex from mri. NeuroImage, 12:340-356, 2000 .

[13] L. Shen and M.K. Chung. Large-scale modeling of parametric surfaces using spherical harmonics. In Third International Symposium on 3D Data Processing, Visualization and Transmission (3DPVT).

[14] M. Styner, I. Oguz, S. Xu, C. Brechbuhler, D. Pantazis, J. Levitt, M. Shenton, and G. Gerig. Framework for the statistical shape analysis of brain structures using spharm-pdm. In Insight Journal, Special Edition on the Open Science Workshop at MICCAI, 2006.

[15] K.J. Worsley, S. Marrett, P. Neelin, A.C. Vandal, K.J. Friston, and A.C. Evans. A unified statistical approach for determining significant signals in images of cerebral activation. Human Brain Mapping, 4:58-73, 1996. 